\title{
拓展浓度加和模型预测三种三嗪类除草剂混合物的时间依赖毒性
}

\author{
王猛超＼cjkstart刘树深* 陈浮 \\ (同济大学环境科学与工程学院长江水环境教育部重点实验室 上海 200092)
}

\begin{abstract}
摘要 浓度加和(CA)模型是评估与预测化学混合物毒性效应的常用模型之一, 已广泛应用于药物组合与农药混配等多 个混合物体系. 然而, CA 不能用于包含不同时间具有不同效应组分的混合物体系, 需要拓展. 以具有不同时间依赖毒 性特征的三种三嗪类除草剂即嗪草䣯(MET)、西草净(SIM)和环嗪酮(HEX)为混合物组分，以直接均分射线法(EquRay) 构建它们的二元混合物, 以青海弧菌 Q67 为受试生物, 在 6 个不同时间(即 $0.25,2,4,8,12$ 和 $16 \mathrm{~h}$ )下测定各混合物组分 及其二元混合物暴露于 Q67 时的发光抑制毒性, 分析与归纳抑制毒性随时间的变化规律. 结果表明, 三种除草剂对发 光菌 Q67 的发光抑制毒性具有不同的时间变化规律: MET 的毒性随时间延长有明显增大的趋势, SIM 的毒性在不同时 间没有显著性变化，而 HEX 随着时间的延长毒性开始有所增加，而后变化很小; 二元混合物毒性的时间变化规律与混 合物组分有关, MET 与 HEX 的二元混合物体系的毒性随暴露时间的延长而逐步增加, MET-SIM 体系开始有所下降而后 缓慢增加，而 SIM-HEX 体系的毒性在不同时间差异不明显; 应用拓展后的 CA 模型分析所有二元混合物在不同时间点 的毒性变化, 表明都是加和的, 没有协同或拮抗作用.
\end{abstract}

关键词＼cjkstart浓度加和; 化学混合物; 直接均分射线; 三溙类除草剂; 时间依赖毒性

\section{Predicting the Time-dependent Toxicities of Three Triazine Herbicide Mixtures to $V$. qinghaiensis sp. Q67 Using the Extended Concentration Addition Model}

\author{
Wang, Mengchao Liu, Shushen* Chen, Fu \\ (Key Laboratory of Yangtze River Water Environment, Ministry of Education, College of Environmental Science and \\ Engineering, Tongji University, Shanghai 200092)
}

\begin{abstract}
The concentration addition (CA) model is a common model for the evaluation and prediction of mixture toxicity and has been widely used in many mixture systems such as drug combination and pesticide mixtures. However, it is necessary to extend the CA so that it is applicable in the mixture systems including components with different effects in different times. In this paper, three triazine herbicides, metribuzin (MET), simetryn (SIM) and hexazinone (HEX), which have different characteristics of the time-dependent toxicity, were selected as mixture components and their binary mixtures were designed by using the direct equipartition ray design (EquRay). Taking $V$. qinghaiensis sp.-Q67 as test organism and 96-well microplate as exposure experiment carrier, the luminescence inhibition toxicities of three triazine herbicides and their binary mixtures were determined at six different time points $(0.25,2,4,8,12$ and $16 \mathrm{~h})$. The fluctuation tendency of the luminescence inhibition toxicity varying with time was analyzed. The results show that the inhibition toxicities of three triazine herbicides to $V$. qinghaiensis sp.-Q67 have different characteristics of the time-dependent toxicity, i.e., the toxicity of MET apparently increases over time, while that of SIM is basically a constant in the range of testing time, but that of HEX first increases with time at the beginning stage and then is basically unchanged. Also, the toxicities of the binary mixtures consisting of three herbicides depend on not only the composition of the mixtures but also the concentration ratio of various components. The toxicity of binary mixture consisting of MET and HEX gradually increases over time, and that of binary MET-SIM mixture at first decreases and then slowly increases, while the toxicity change is not significant in the SIM-HEX mixture system. Moreover, all the toxicities of binary mixtures can be effectively predicted by the CA model which had been extended by regarding the concentration of no-effect component as infinity, being additive, not synergistic or antagonistic. Keywords concentration addition; chemical mixtures; EquRay; triazine herbicides; time-dependent toxicity
\end{abstract}

\footnotetext{
*E-mail: ssliuhl@263.net

Received October 8, 2013; published November 29, 2013.

Supporting information for this article is available free of charge via the Internet at http://sioc-journal.cn. gram of Higher Education (No. 20120072110052).

项目受国家自然科学基金(Nos. 21177097, 20977065)和高等学校博士学科点专项科研基金(No. 20120072110052)资助.
}

Project supported by the National Natural Science Foundation of China (Nos. 21177097, 20977065) and the Specialized Research Fund for the Doctoral Pro- 


\section{1 引言}

化学混合物普遍存在于各种环境介质中, 从单个化 学组分的浓度-效应信息预测不同浓度组成混合物的毒 性效应, 进而建立相应评估与预测模型, 是目前化学混 合物研究的流行方法. 大量事实表明, 浓度加和 (concentration addition, 简称 CA) 模型是研究最多也是 应用最广泛的化学混合物评估与预测模型之一 ${ }^{[1 \sim 5]}$. CA 模型已成功地应用于多个药物组合与农药混配体系的 混合物效应评估 ${ }^{[6 \sim 10]}$. 近年来, 发现有些环境污染物的 急性与慢性毒性显著不同, 有些污染物急慢性毒性变化 不大, 而有些污染物的毒性随时间的变化而变化. 例如, Zhu 等 ${ }^{[11]}$ 在研究六种三嗪除草剂对发光菌的长期和短期 毒性后发现, DES (Desmetryne)和 SIM (Simetryn)的长短 期毒性没有显著差异, VEL (Velpar)、MET (Metribuzin) 和 PRO (prometon)的长期毒性明显大于短期毒性, 而 AMI (Aminotriazine) 只有长期毒性. 在多个关于杀虫剂 与抗生素短期与长期毒性的比较研究中发现了类似的 规律 ${ }^{[12,13]}$. Froehner 等 ${ }^{[14,15]}$ 在短长期毒性之间, 增加多个 测试时间点, 考察了污染物毒性随时间的变化规律, 发 现污染物毒性随时间的变化并非全是单调的, 在二硝基 邻甲酚和 2,4,5-三氯酚对费氏弧菌的暴露实验中, 随着 暴露时间的延长甚至出现了低剂量刺激高剂量抑制的 hormesis 现象. 为方便, 把污染物具有的短期与长期或 不同时间之间的毒性差异称为时间依赖毒性或简称时 间毒性. 目前, 由具有不同时间毒性特征的污染物构成 的混合物其毒性效应如何评估与预测的问题尚缺少方 法与案例.

最近, Rodney 等 ${ }^{[16]}$ 在评述了大量农药混合物的毒性 效应文献之后得出结论, 绝大多数农药混合物的毒性效 应都是加和的, 即都可用 CA 模型进行评估与预测. 然 而, 农药混合物体系是一个非常复杂的体系, 现有研究 结果特别是多元混合物的结果往往只研究这个体系中 某一个特殊的混合物的某一个混合物点, 比如等毒性浓 度比混合物的 $\mathrm{EC}_{50}$, 这既不是实际环境体系中的代表性 混合物也不能表征整个混合物体系 ${ }^{[17]}$. 另一方面, 由 $\mathrm{CA}$ 模型可知, 在混合物效应为 $\mathrm{x}$ 时, 只有当各个组分单 独存在下能产生同样的效应时模型才能成立. 而具有不 同时间依赖毒性特征的污染物, 比如某个污染物没有急 性毒性(不存在 $x$ ), 这时 CA 模型不能应用, 必须进行适 当改造或拓展.

为此, 本文选择具有不同时间依赖毒性特征的嗪草 酮 MET(毒性随时间增加而显著增加)、西草净 SIM(毒 性随时间变化不明显)和环嗪酮 HEX(开始毒性随时间增 加而后基本不变)为混合物组分, 应用直接均分射线法 (EquRay) ${ }^{[18]}$ 设计二元混合物, 在不同暴露时间下测定这 些除草剂及其二元混合物对青海弧菌 Q67 的发光抑制 毒性, 改造 CA 模型使之能应用于这些混合物的毒性评 估与预测, 为具有不同时间依赖特征污染物的混合物毒
性评估与预测提供方法途径与应用实例.

\section{2 结果与讨论}

\section{1 化学混合物的表征}

混合物毒性具有组成与浓度依赖性 ${ }^{[16]}$. 具有一定组 成的化学混合物是一个复杂的系统, 其中包含无数个组 成一定而各组分浓度各异的混合物, 有必要首先对这些 混合物进行合适表征.一个混合物组分(Component)可 以通过测量剂量-效应曲线 $(\mathrm{CRC})$ 来表征该组分的毒性 特征从而获得某些特征浓度如半效应浓度 $\left(\mathrm{EC}_{50}\right)$ 和无观 测效应浓度(NOEC) 等. 为了获得类似单个组分的混合 物 $\mathrm{CRC}$, 可采用固定各组分在混合物中的浓度分数(也 称浓度比)并逐步改变混合物总浓度的方法(逐渐稀释) 设计一系列混合物并测试其毒性效应，进而构建混合物 的 CRC. 这些混合物(点)在由各组分张成的浓度空间中 分布在一条从原点出发的射线上，把这些混合物统称为 混合物射线. 为了系统表征化学混合物, 称具有一定组 成的一系列混合物为混合物体系, 如二元混合物与三元 混合物等都是混合物体系. 混合物体系中的一系列具有 固定浓度分数的混合物称为混合物射线(1 条混合物射 线可构成混合物的 (RC). 射线上的某个点其各组分具 有确定的浓度是某个具体的混合物. 这样, 本文的三种 除草剂构成的三元混合物是体系. 为了全面考虑组成依 赖性, 其中两两组合构成的二元混合物也是体系. 为考 察混合物的浓度依赖性又从每个二元混合物体系中优 选了 3 条射线(图 1).

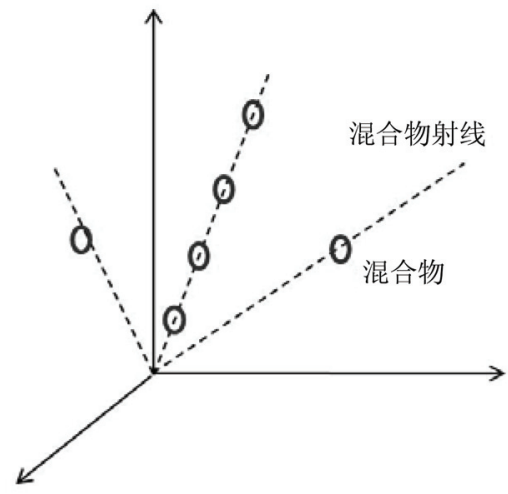

图 1 混合物体系浓度空间表征

Figure 1 Mixture system in concentration space

图 1 代表混合物体系, 其中的 3 条虚线是 3 条混合 物射线，而虚线上的点(圆圈)是具有确定浓度的具体混 合物.

\section{2 除草剂毒性随时间的变化}

三种除草剂在不同测试时间内的浓度-效应数据都 可以用两参数(位置参数 $\alpha$ 和形状参数 $\beta$ ) 非线性 Logit 函 数进行有效表征. 位置参数 $\alpha$ 和形状参数 $\beta$ 、拟合相关 
系数 $R$ 与均方根误差 $\mathrm{RMSE}$ 和两个特征浓度 $\left(\mathrm{EC}_{20}\right.$ 与 $\mathrm{EC}_{50}$ ) 及其 $95 \%$ 置信区间参见支持材料表 $\mathrm{S} 1.6$ 个测试时 间下的 CRC 绘于支持材料图 S1 中. 三种除草剂的毒性 效应随时间的变化曲线如图 2 所示. 可知, 除草剂对 Q67 的发光抑制毒性随时间的变化规律不同.

对于 $\mathrm{MET}$, 如果以 $\mathrm{pEC}_{50}$ 为毒性指标, 开始 3 个时 间点几乎没有毒性, $\mathrm{pEC}_{50}$ 可视为 $0,8 \mathrm{~h}$ 开始产生毒性, 而后毒性随暴露时间延长而增大. 应该注意, $\mathrm{pEC}_{50}$ 在开 始 3 个时间点以及 $\mathrm{pEC}_{20}$ 在开始 2 个时间点虽然都视为 0 , 但实际上在 0.25 和 $2 \mathrm{~h}$ 也观测到约 $15 \% \sim 20 \%$ 的发光 抑制率. $\mathrm{SIM}$ 的 $\mathrm{pEC}_{20}$ 和 $\mathrm{pEC}_{50}$ 波动范围很小, 毒性不随 时间而显著变化. HEX 的毒性(无论 $\mathrm{pEC}_{20}$ 或 $\mathrm{pEC}_{50}$ ) 开始 随着时间的延长有所增加, 而后基本不变. 这与嗪草酩 MET、西草净 SIM 和环嗪酮 HEX 的急性暴露(15 min) 和长期暴露 $(12 \mathrm{~h})$ 的结果是一致的 ${ }^{[11]}$.

\section{3 二元混合物时间毒性特征}

通过直接均分射线法(EquRay) ${ }^{[18]}$ 对每个二元混合 物体系(MET-SIM, MET-HEX 或 SIM-HEX)设计 3 条射 线(各射线中组分的浓度分数或浓度比参见表 1), 每条 射线安排 12 个混合物点, 各个混合物(点)在不同时间下 测定其对 Q67 的发光抑制毒性. 结果表明, 每条射线(共 9 条)的浓度-效应数据也都可用 Logit 函数有效拟合. 各 条射线拟合所得的位置参数 $\alpha$ 和形状参数 $\beta$ 、拟合相关 系数 $R$ 与均方根误差 $\mathrm{RMSE}$ 和两个特征浓度 $\left(\mathrm{EC}_{20}\right.$ 与 $\mathrm{EC}_{50}$ )及其 $95 \%$ 置信区间参见支持材料表 $\mathrm{S} 2.6$ 个测试时 间下的各射线 $\mathrm{CRC}$ 绘于支持材料图 $\mathrm{S} 2$ 中. 为了和单个 纯组分进行比较, 同样选择 $\mathrm{pEC}_{20}$ 和 $\mathrm{pEC}_{50}$ 为两个毒性 指标, 制作其随时间的变化图, 考察各个体系的 3 条混 合物射线毒性随时间的变化规律，结果见图 3 .

结果表明, 含有 MET 的混合物体系如 MET-SIM 和 MET-HEX 体系的所有混合物射线的毒性随时间的延长 都有一定的变化, 而不含 MET 组分的混合物 SIM-HEX 体系中的混合物毒性随时间的变化不显著. MET-SIM二 元体系的 3 条射线(R1, R2 和 R3)中, 混合物毒性在前 4 个时间点甚至有所下降(R2 和 R3), 后 3 个时间点, 其毒 性随时间延长缓慢增加. 例如，射线 $\mathrm{R} 1$ 的 $\mathrm{pEC}_{50}$ 在 8,12 和 $16 \mathrm{~h}$ 时分别为 $3.0,3.5$ 和 3.9. $\mathrm{pEC}_{20}$ 分别为 $3.4,4.1$ 和
4.4. MET-HEX 二元混合物体系中 3 条射线的毒性变化 从 4 到 $16 \mathrm{~h}$ 有增加的趋势, 特别在 MET 浓度分数较大 的情况下增加比较明显, 如图 3 中 R1 比 R2 变化明显, 而 R3 变化不大. 而 SIM-HEX 体系的 3 条射线其毒性变 化基本不变, 特别在 HEX 的浓度分数高的射线(如 R3) 中. 这说明混合物组分除草剂在二元混合物体系中的毒 性变化规律与单独存在时的毒性变化规律一致, 暗示它 们的混合物毒性具有加和性，没有协同或拮抗.

表 19 条混合物射线各组分浓度比 $\left(p_{\mathrm{i}}\right)$ 及储备液浓度

Table 1 Concentration ratios $\left(p_{\mathrm{i}}\right)$ of various components and the concentrations of stocks for nine mixture rays

\begin{tabular}{llcccc}
\hline \multirow{2}{*}{ No. } & Mixture ray & \multicolumn{3}{c}{$p_{\mathrm{i}} / \%$} & \multirow{2}{*}{ Stock $/\left(\mathrm{mol} \cdot \mathrm{L}^{-1}\right)$} \\
\cline { 3 - 5 } & & MET & SIM & HEX & \\
\hline 1 & MET-SIM-R1 & 83.87 & 16.13 & & $2.67 \mathrm{E}-03$ \\
2 & MET-SIM-R2 & 63.42 & 36.58 & & $2.40 \mathrm{E}-03$ \\
3 & MET-SIM-R3 & 36.62 & 63.38 & & $2.12 \mathrm{E}-03$ \\
4 & MET-HEX-R1 & 65.98 & & 34.02 & $3.75 \mathrm{E}-03$ \\
5 & MET-HEX-R2 & 39.27 & & 60.73 & $4.81 \mathrm{E}-03$ \\
6 & MET-HEX-R3 & 17.73 & & 82.27 & $6.23 \mathrm{E}-03$ \\
7 & SIM-HEX-R1 & & 78.18 & 21.82 & $2.20 \mathrm{E}-03$ \\
8 & SIM-HEX-R2 & & 54.43 & 45.58 & $2.83 \mathrm{E}-03$ \\
9 & SIM-HEX-R3 & & 28.47 & 71.53 & $4.12 \mathrm{E}-03$ \\
\hline
\end{tabular}

前已证明，西草净(SIM)的毒性几乎不随暴露时间 而变化, 环嗪酮(HEX)的毒性只在 $4 \mathrm{~h}$ 之前有变化, 而溙 草酮 (MET)的毒性随暴露时间的延长而增加. 所以, SIM-HEX 二元混合物体系在不同时间没有显著性时间 毒性差异. 在 MET-SIM 二元体系中, 当 MET 的浓度比 例足够高的时候, 其混合物射线的毒性就发生变化, 如 MET-SIM-R1. 在 MET-HEX 二元体系中, 混合物的时 间毒性变化与 MET 有着相似的规律, 它们的毒性随暴 露时间的延长而逐步增加, 尽管该体系中 MET 的浓度 分数 $\left(p_{\mathrm{i}}\right)$ 低于 MET-SIM 体系中的 $p_{\mathrm{i}}$, 但是其在 METHEX 中的实际浓度更高; 另一方面, HEX 对 Q67 的毒性 比 SIM 小, 结合考虑 SIM 和 HEX 在各自体系中的实际 浓度, 发现 HEX 贡献的毒性效应比 SIM 贡献的毒性效 应小，而 MET 在 MET-HEX 具有更大的影响. 因此，混
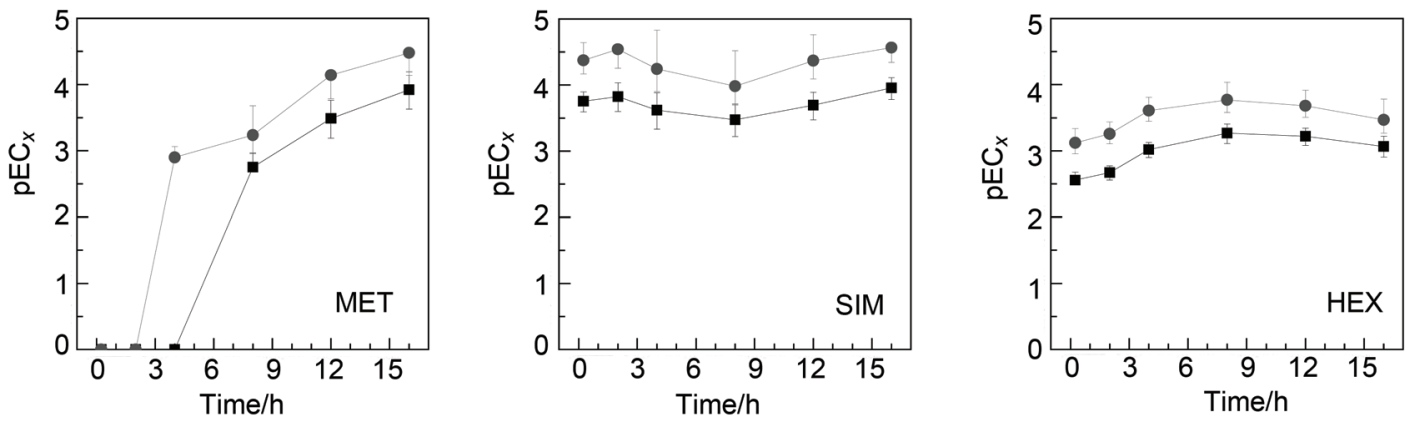

图 2 三种三嗪类除草剂毒性随时间的变化曲线 $\left(\mathbf{m :} \mathrm{pEC}_{50} ; \bullet: \mathrm{pEC}_{20}\right)$

Figure 2 Plot of toxicity $\left(\mathrm{pEC}_{x}\right)$ of three triazine herbicides versus time where $\boldsymbol{m}$ and $\bullet$ refer to $\mathrm{pEC}_{50}$ and $\mathrm{pEC}_{20}$, respectively 

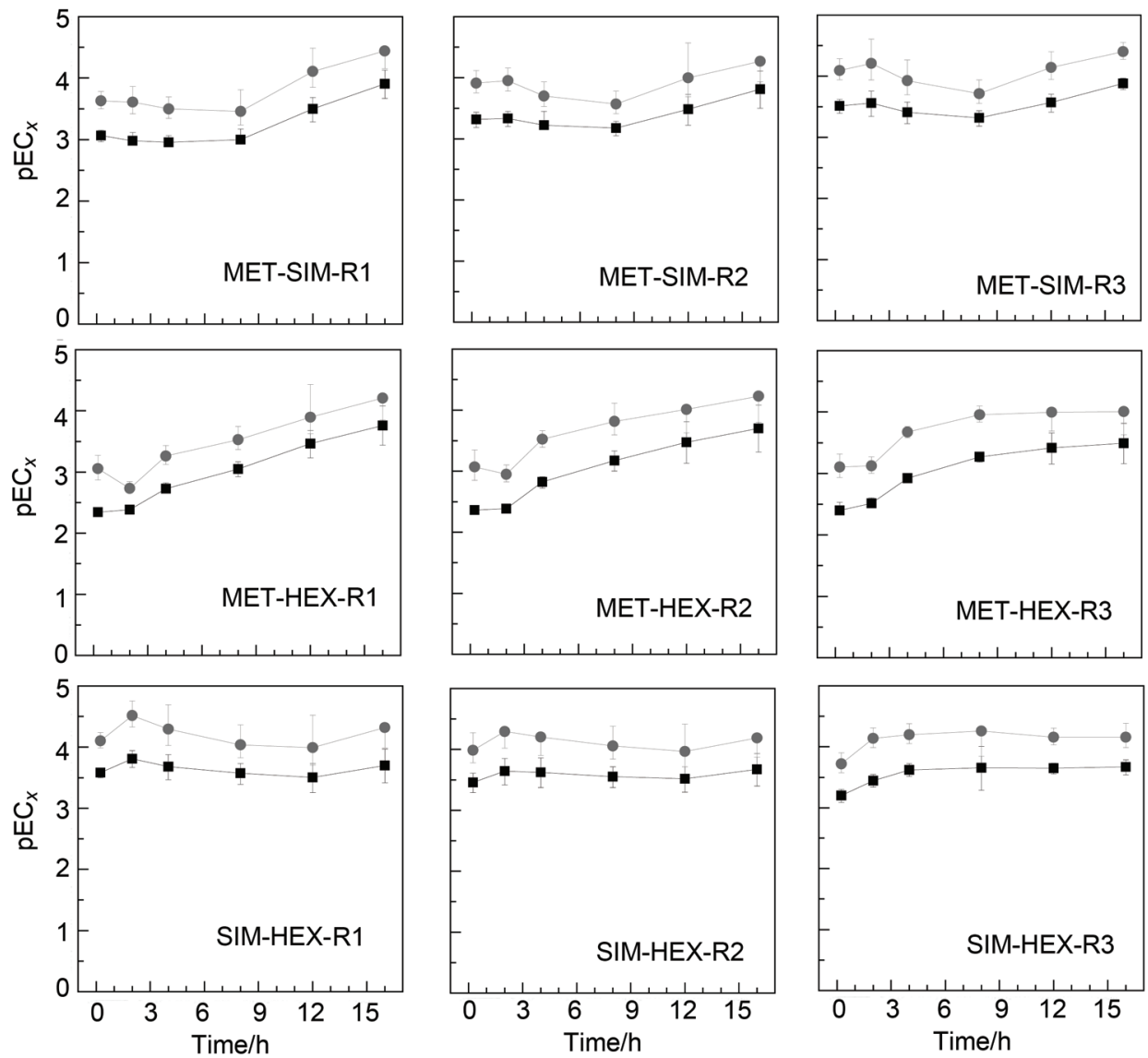

图 39 条混合物射线的毒性随时间的变化曲线 $\left(\boldsymbol{\bullet}: \mathrm{pEC}_{50} ; \bullet: \mathrm{pEC}_{20}\right)$

Figure 3 Plot of toxicities, $\mathrm{pEC}_{50}(\boldsymbol{\bullet})$ and $\mathrm{pEC}_{20}(\bullet)$, of nine mixture rays versus time

合物的时间毒性特征不仅与有时间毒性差异组分的浓 度分数有关, 还与无时间毒性差异组分实际贡献的毒性 效应大小有关.

\section{4 二元混合物时间毒性的可预测性}

由 2.3 节的分析可知, 二元混合物体系可能具有加 和毒性效应. 应用拓展的浓度加和(CA)模型评估 3 个二 元混合物体系中所有 9 条混合物射线的毒性效应(参见 支持材料图 S2). 结果表明, 9 条射线在各个时间下 $(0.25$, $2,4,8,12$ 和 $16 \mathrm{~h}$ ) 拓展 CA 模型预测的 CRC 曲线全部位 于实验测试 CRC 的置信区间范围内, 说明混合物射线 的毒性都可用 CA 模型进行预测. 或者说, 混合物毒性 是浓度加和的, 没有毒性相互作用, 加和性也不随暴露 时间的变化而变化. 即对于所研究的三种除草剂的二元 混合物, 其毒性的加和性既没有时间(不同时间)依赖性, 也没有浓度比(不同射线)和浓度 (CRC 上不同点)依赖性. 这与 Rodney 等 ${ }^{[16]}$ 关于农药混合物之间除了极少数例外 基本不存在相互作用而可用 CA 模型预测的结论一致.

应该指出, 由于嗪草酮(MET) 在 $0.25,2,4 \mathrm{~h}$ 下几乎 没有毒性, 在这些时间点的二元混合物(MET-SIM 和 MET-HEX)射线不能直接应用 CA 模型评估混合物毒性. 在加和条件下, 1 个组分完全没有毒性效应的二元混合
物, 其毒性完全由另一个组分贡献, 这时可认为无毒性 组分的效应浓度 $\mathrm{EC}_{x}$ 为无穷大, 将这个 $\mathrm{EC}_{x}$ 代入浓度加 和模型, 就可以利用 $\mathrm{CA}$ 模型进行预测了.

\section{3 结论}

本文以三种对青海弧菌 Q67 具有不同时间毒性特 征的除草剂即嗪草酮(明显时间毒性差异)、西草净(不随 时间变化)和环嗪酮(开始随时间增加而后变化很小)为 混合物组分，通过直接均分射线法选择混合物体系中 3 个代表性射线, 应用拓展浓度加和模型, 证明了所研究 除草剂混合物的毒性是浓度加和的，其加和性没有时 间、组分浓度比以及组分浓度依赖性.

\section{4 实验部分}

\section{1 仪器与试剂}

PowerWave 微孔板分光光度计(美国 BIO-TEK 公 司)、150C 型恒温振荡培养箱(金坛市亿通电子有限公 司)、BT25S 型五位电子天平(赛多利斯科学仪器(北京) 有限公司)、SW-CJ-IF 超净工作台(苏州佳宝净化工程设 备有限公司)、Milli-Q 超纯水系统(美国 Millipore 公司)、 
Eppendorf 单道可调移液器 $(10 \sim 100 \mu \mathrm{L})$ (德国 Eppendorf 公司)和手动 12 道移液器(L12-200) (美国 Rainin 公司).

嗪草酩(MET)、西草净(SIM)、环嗪酩(HEX)均为优 级纯试剂(基本信息参见支持材料表 $\mathrm{S} 3$, 结构式见支持 材料图 S3), 均购自德国 Dr.ehrenstorfer 公司. 试验前用 Milli-Q 超纯水配制储备液并保存在棕色瓶中, 置于 4 ${ }^{\circ} \mathrm{C}$ 冰箱保存待用.

\section{2 菌种与培养}

青海弧菌 Q67 ( $V$. qinghaiensis sp. Q67)购自北京滨 松光子技术股份有限公司, 液体培养基配方及细菌培养 方法参见文献 ${ }^{[19]}$ ，浓缩培养基 ${ }^{[11]}$ 除了胰蛋白炼 $6.63 \mathrm{~g}$, 酵母浸出膏 $8.78 \mathrm{~g}$, 甘油 $6.90 \mathrm{~g}$ 之外, 其它成分的质量 是液体培养基相应成分质量的 2 倍(1000 mL 蒸馏水). 用 $1 \mathrm{~mol} / \mathrm{L} \mathrm{NaOH}$ 或 $\mathrm{HCl}$ 调整培养基 $\mathrm{pH}$ 值至 8.5 9.0, 分装于 $50 \mathrm{~mL}$ 锥形瓶中, 每瓶各约 $30 \mathrm{~mL}$, 用牛皮纸包 扎并用橡皮筋扎紧雉形瓶瓶口, 经 $121{ }^{\circ} \mathrm{C}$ 高压蒸汽灭 菌 $20 \mathrm{~min}$, 冷却后于 $4{ }^{\circ} \mathrm{C}$ 冰箱保存备用.

\section{3 不同时间的发光抑制毒性测试}

选用白色不透明 96 孔标准板(8 行 12 列)作为测试 载体. 为了避免边缘效应 ${ }^{[20]}, 96$ 孔微板周围的 36 个孔分 别加入 $200 \mu \mathrm{L}$ 超纯水. 余下 60 个孔总体积 $200 \mu \mathrm{L}$, 其 中含菌液 $100 \mu \mathrm{L}$. 这 60 个孔安排 24 个平行空白(对照), 12 个浓度梯度(处理)各 3 次平行, 对照与处理在微板上 的分布按文献 ${ }^{[21]}$ 中的图 3 设计. 设计好的微板送入(22 $\pm 1){ }^{\circ} \mathrm{C}$ 的 PowerWave 微孔板分光光度计 (发光检测仪) 样品室, 分别在 6 个暴露时间点, 即 $0.25,2,4,8,12$ 和 $16 \mathrm{~h}$ 下, 读取各孔相对发光单位(RLU)值. 每板至少重 复 3 次. 发光抑制毒性表达为混合物组分或其混合物暴 露 RLU 对空白 RLU 的抑制百分率. 与文献[19, 21]不同, 这里加入的菌液称为时间菌液 ${ }^{[22]}$, 即指先将青海弧菌 在液体培养基中培养至对数生长期(发光数 $\geqslant 3 \times 10^{6}$ ), 取一定量的青海弧菌菌液, 与等体积的浓缩培养基混 合, 之后放入 $22{ }^{\circ} \mathrm{C}$ 培养箱中进行预培养 $0.5 \sim 1 \mathrm{~h}$.

\section{4 各种二元混合物设计}

对于具有一定组成的每一个二元混合物体系, 应用 直接均分射线(EquRay)法 ${ }^{[18]}$ 设计 3 条混合物射线(R1, $\mathrm{R} 2$ 和 R3), 同一条射线中某组分的浓度分数或浓度比 $\left(p_{\mathrm{i}}\right)$ 即该组分浓度所占混合物总浓度的分数保持不变. 三种除草剂(MET, SIM 和 HEX) 可构成 3 个二元混合物 体系(MET-SIM, MET-HEX 和 SIM-HEX), 对各体系设 计的 9 条混合物射线中各组分浓度分数 $\left(p_{\mathrm{i}}\right)$ 及储备液 (Stock)如表 1 所示. 每条射线安排 12 个具有不同总浓度 的混合物点, 并用 4.3 节方法测试各个混合物点对青海 弧菌在 6 个不同时间下的发光抑制毒性.

\section{5 浓度-效应曲线的拟合与毒性预测}

对实验获得的单个除草剂与各混合物射线的浓度效应(发光抑制毒性)数据, 应用 2 参数(位置参数 $\alpha$ 和形 状参数 $\beta$ ) 的 Logit 函数进行最佳非线性回归 ${ }^{[21]}$, 并求 $95 \%$ 的观测置信区间 ${ }^{[23]}$.

采用浓度加和 $(\mathrm{CA})$ 模型为加和性参考标准, 当混合 物实验毒性大于 $\mathrm{CA}$ 模型预测的毒性时称为协同，而当 实验毒性小于预测毒性时称为拮抗 ${ }^{[17]}$.

对于其中一个组分无毒性的二元混合物，其毒性完 全由该混合物中的另一个有毒性组分决定，这时可认为 无毒性组分的效应浓度 $\mathrm{EC}_{x}$ 为无穷大.

\section{References}

[1] Baylay, A. J.; Spurgeon, D. J.; Svendsen, C.; Griffin, J. L.; Swain, S C.; Sturzenbaum, S. R.; Jones, O. A. H. Ecotoxicology 2012, 21, 1436.

[2] Howard, G. J.; Schlezinger, J. J.; Hahn, M. E.; Webster, T. F. Environ. Health Perspect. 2010, 118, 666.

[3] Backhaus, T.; Faust, M.; Scholze, M.; Gramatica, P.; Vighi, M.; Grimme, L. H. Environ. Toxicol. Chem. 2004, 23, 258.

[4] Faust, M.; Altenburger, R.; Backhaus, T.; Blanck, H.; Boedeker, W.; Gramatica, P.; Hamer, V.; Scholze, M.; Vighi, M.; Grimme, L. H. Aquat. Toxicol. 2001, 56, 13.

[5] Gregorio, V.; Chevre, N.; Junghans, M. Environ. Toxicol. Chem. 2013, 32, 2387.

[6] Jacobsen, N. W.; Brooks, B. W.; Halling-Sorensen, B. Regul. Toxicol. Pharmacol. 2012, 62, 441.

[7] Thienpont, B.; Barata, C.; Raldua, D. Toxicol. Appl. Pharmacol. 2013, 269, 169

[8] Liu, S. S.; Wang, C. L.; Zhang, J.; Zhu, X. W.; Li, W. Y. Ecotoxicol. Environ. Safety 2013, 95, 98.

[9] Hernandez, A. F.; Parron, T.; Tsatsakis, A. M.; Requena, M.; Alarcon, R.; Lopez-Guarnido, O. Toxicology 2013, 307, 136.

[10] Christen, V.; Crettaz, P.; Oberli-Schraemmli, A.; Fent, K. Toxicol. Appl. Pharmacol. 2012, 259, 169.

[11] Zhu, X. W.; Liu, S. S.; Ge, H. L.; Liu, Y. Water Res. 2009, 43, 1731.

[12] Backhaus, T.; Grimme, L. H. Chemosphere 1999, 38, 3291.

[13] Zhu, X. W.; Liu, S. S.; Zhang, Q.; Liu, Y. Res. Environ. Sci. 2009, 22, 589. (朱祥伟, 刘树深, 张琼, 刘㘿, 环境科学研究, 2009, 22, 589.)

[14] Froehner, K.; Meyer, W.; Grimme, L. H. Chemosphere 2002, 46, 987.

[15] Cai, X. Y.; Ye, J.; Sheng, G. Y.; Liu, W. P. Environ. Sci. Pollut. Res. 2009, 16, 459 .

[16] Rodney, S. I.; Teed, R. S.; Moore, D. R. J. Hum. Ecol. Risk. Assess. 2013, 19, 1557.

[17] Liu, S. S.; Liu, L.; Chen, F. Acta Chim. Sinica 2013, 71, 1335. (刘树 深, 刘玲, 陈浮, 化学学报, 2013, 71, 1335.)

[18] Dou, R. N.; Liu, S. S.; Mo, L. Y.; Liu, H. L.; Deng, F. C. Environ. Sci. Pollut. Res. 2011, 18, 734.

[19] Liu, S. S.; Song, X. Q.; Liu, H. L.; Zhang, Y. H.; Zhang, J. Chemosphere 2009, 75, 381 .

[20] Wsol, V.; Fell, A. F. J. Biochem. Bioph. Methods 2002, 54, 377.

[21] Liu, S. S.; Zhang, J.; Zhang, Y. H.; Qin, L. T. Acta Chim. Sinica 2012, 70, 1151. (刘树深, 张瑾, 张亚辉, 覃礼堂, 化学学报, 2012, 70, 1151.)

[22] Zhang, J.; Liu, S. S.; Yu, Z. Y.; Zhang, J. Chemosphere 2013, 91, 462.

[23] Zhu, X. W.; Liu, S. S.; Ge, H. L.; Liu, Y. China Environ. Sci. 2009, 29, 113. (朱祥伟, 刘树深, 葛会林, 刘堰, 中国环境科学, 2009, 29, 113.) 